九州大学学術情報リポジトリ

Kyushu University Institutional Repository

\title{
Herbicidal Activity of Caprolactam Derivatives
}

Aikawa, Takatoshi

Laboratory of Pesticide Chemistry, Faculty of Agriculture, Kyushu University

Oshima, Yasuyoshi

Laboratory of Pesticide Chemistry, Faculty of Agriculture, Kyushu University

Eto, Morifusa

Laboratory of Pesticide Chemistry, Faculty of Agriculture, Kyushu University

https://doi.org/10.5109/22889

出版情報 : 九州大学大学院農学研究院紀要. 20 (2)，pp.75-78，1976-02. Kyushu University バージョン：

権利関係 : 
J. Fac. Agr., Kyushu Univ., 20, 75-78 (1976)

\title{
Herbicidal Activity of Caprolactam Derivatives
}

\author{
Takatoshi Aikawa, Yasuyoshi Oshima and \\ Morifusa Eto \\ Laboratory of Pesticide Chemistry, Faculty of Agriculture, \\ Kyushu University, Fukuoka
}

(Received August 28, 1975)

\begin{abstract}
Many derivatives of $\varepsilon$-caprolactam were prepared and examined for herbicidal activity. Some $N$-alkyl derivatives showed considerable herbicidal activity by pre-emergence treatment.
\end{abstract}

Several commercial herbicides have the $-\mathrm{CO} \cdot \mathrm{N}<$ group in the molecule as amides, carbamates, and ureas, for example, $N, N$-diallylchloroacetamide (allidochlor, CDAA), isopropyl $\mathrm{N}$-m-chlorophenylcarbamate (chlorpropham, IPC), and 1(3,4-dichlorophenyl)-3,3-dimethylurea (diuron, DCMU). However, only a few reports have been published on the physiological activity of lactams towards plants, although some lactams were patented as herbicides (Wilson et al., 1964). Since $\varepsilon$-caprolactam is available in a great quantity, it is a convenient starting material for the study of structure-activity relationship of lactam derivatives. This paper deals with the herbicidal activity of some derivatives of $\varepsilon$-caprolactam.

\section{MATERIALS AND METHODS}

\section{Synthesis}

All derivatives of $\varepsilon$-caprolactam were synthesized according to reported methods, as briefly described below, and purified by distillation or recrystallization.

$N$-Alkylcaprolactams (Compd. 4-16) were prepared by the reaction of appropriate haloalkane with the sodium salt of $\varepsilon$-caprolactam which was obtained by the interaction of sodium and the lactam (Marvel and Moyer, 1957).

$N$-Acylcaprolactams (Compd. 17-23) were synthesized by the reaction of acyl chlorides or anhydrides with $\varepsilon$-caprolactam in the presence of dimethylaniline or triethylamine. Chlorination of caprolactam was performed according to the procedure of Wineman et al. (1958); $\varepsilon$-caprolactam was treated with chlorine in the presence of phosphorus pentachloride to yield 3,3-dichlorohexahydro- $2 \mathrm{H}$ azepin-2-one (Compd. 3). The dichloro derivative was converted further to the monochloro derivative (Compd. 2) by catalytic hydrogenation in the presence of paladium-charcoal.

Caprolactims and amidine derivatives were prepared according to the method of Benson and Cairus (1948). $\varepsilon$-Caprolactam reacted with a dialkyl sulfate to produce the corresponding $\boldsymbol{O}$-alkylcaprolactim (Compd. 30,31 ). The imino 
ether was heated with an appropriate amine to give corresponding amidine [7( $\mathrm{N}$-substituted) aminotetrahydro-2H-azepine] (Compd. 24-29).

\section{Assay for herbicidal activity}

Pre-emergence herbicidal activity was examined in Petri dishes. An acetone solution of a test compound was poured on two sheets of filter paper placed on the bottom of a Petri dish. After complete evaporation of the solvent, $5 \mathrm{ml}$ of distilled water was poured into the dish. The concentrations tested were 10000,1000 , and $500 \mathrm{ppm}$. On the filter paper of each dish 15 seeds of rice or radish or 10 seeds of azuki-bean were placed and kept with a cover in a dark room at $25^{\circ} \mathrm{C}$ for germination. After 3 days the seedlings were cultured under natural light for 1 week. Evaluation of herbicidal activity was performed by examining the inhibition rate in germination and growth. The herbicidal ratings were determined for each plant species according to the following scale.

$+:$ stimulation of germination

0 : no inhibition on germination and seedling growth

$1: 30 \%$ or lower inhibition

$2: 30-60 \%$ inhibition

$3: 60 \%$ or higher inhibition

4 : complete inhibition

\section{RESULTS AND DISCUSSION}

Thirty derivatives were prepared from $\varepsilon$-caprolactam. They include $N$-alkyl, $N$-acyl, $\alpha$-halo, $O$-alkyl, and amidine derivatives. Their herbicidal activity was examined and the results are shown in Tables 1,2 , and 3.

Although $\varepsilon$-caprolactam (Compd. 1) had an activity stimulating the germination and growth of Pisum sativum at low concentrations (100-0.1 ppm) (Baszynski and Rejniak, 1958), it inhibited germination of all tested plants at 10000 ppm. The inhibitory activity increased by modification of the structure. The activity of caprolactam derivatives decreases in the following order: $N$-alkyl $>$ amidine $>N$-acyl and imino ether derivatives. The imino ethers (Compd. 30, 31) are less active than the parent compound, caprolactam.

In the series of $N$-alkyl derivatives, the herbicidal activity increases with bulkiness of the alkyl group until the optimum. Thus, $N$-isoamylcaprolactam (Compd. 10) was the strongest herbicide and the isobutyl derivative (Compd. 9) was the next in the examined derivatives of $\varepsilon$-caprolactam. Their activity appeared, however, to be somewhat lower than commercial herbicides such as PCP (pentachlorophenol) and 2,4-D (2,4-dichlorophenoxyacetic acid). n-Alkylcaprolactams (Compd. 4-6, 8) were less active and higher $N$-alkyl derivatives (Compd. 11, 12) were almost completely inactive as herbicides. In contrast, $N$. benzyl derivatives (Compd. 13, 14) showed high activity.

$\alpha$-Chlorination caused increase in herbicidal activity. This is particularly effective with $N$-alkylcaprolactams; compare the activity of Compd. 15 and 16 with the corresponding nonchlorinated $N$-alkylcaprolactams (Compd. 4 and 7 ). In the series of $N$-unsubstituted (Compd. 1-3) and $N$-acylated caprolactams (Compd. 17-19), the effect of $\alpha$-chlorination is not so distinctive. 
Table 1. Herbicidal activity of $\alpha$-halo- and $N$-alkyl-caprolactams.

\begin{tabular}{|c|c|c|c|c|c|c|c|c|c|c|c|}
\hline \multirow{2}{*}{$\begin{array}{c}\text { Compd. } \\
\text { No. }\end{array}$} & \multirow[b]{2}{*}{$\mathrm{R}$} & \multirow[b]{2}{*}{$X$} & \multicolumn{3}{|c|}{ Radish } & \multicolumn{3}{|c|}{ Azuki-bean } & \multicolumn{3}{|c|}{ Rice } \\
\hline & & & 100 & 10 & 5 & 100 & 10 & 5 & $\stackrel{100}{\gamma}$ & 10 & 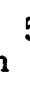 \\
\hline $\begin{array}{r}1 \\
2 \\
3 \\
4 \\
5 \\
6 \\
7 \\
8 \\
9 \\
10 \\
11 \\
12 \\
13 \\
14 \\
15 \\
16\end{array}$ & $\begin{array}{l}\mathrm{H} \\
\mathrm{H} \\
\mathrm{H} \\
\mathrm{CH}_{3} \\
\mathrm{C}_{2} \mathrm{H}_{5} \\
\mathrm{CH}_{2} \mathrm{CH}=\mathrm{CH}_{2} \\
i-\mathrm{C}_{3} \mathrm{H}_{7} \\
n-\mathrm{C}_{4} \mathrm{H}_{9} \\
i-\mathrm{C}_{4} \mathrm{H}_{9} \\
i-\mathrm{C}_{5} \mathrm{H}_{11} \\
n-\mathrm{C}_{8} \mathrm{H}_{17} \\
n-\mathrm{C}_{12} \mathrm{H}_{25} \\
\mathrm{CH}_{2} \mathrm{C}_{6} \mathrm{H}_{5} \\
\mathrm{CH}_{2} \mathrm{C}_{6} \mathrm{H}_{4}-0-\mathrm{Cl} \\
\mathrm{CH}_{3} \mathrm{Cl}_{4} \\
i-\mathrm{C}_{3} \mathrm{H}_{7}\end{array}$ & $\begin{array}{l}\mathrm{H}_{2} \\
\mathrm{Cl}, \mathrm{H} \\
\mathrm{Cl}_{2} \\
\mathrm{H}_{2} \\
\mathrm{H}_{2} \\
\mathrm{H}_{2} \\
\mathrm{H}_{2} \\
\mathrm{H}_{2} \\
\mathrm{H}_{2} \\
\mathrm{H}_{2} \\
\mathrm{H}_{2} \\
\mathrm{H}_{2} \\
\mathrm{H}_{2} \\
\mathrm{H}_{2} \\
\mathrm{Cl}_{2} \\
\mathrm{Cl}_{2}\end{array}$ & $\begin{array}{l}1 \\
4 \\
4 \\
4 \\
4 \\
4 \\
4 \\
4 \\
4 \\
4 \\
0 \\
0 \\
4 \\
4 \\
4 \\
4\end{array}$ & $\begin{array}{l}0 \\
1 \\
1 \\
0 \\
0 \\
0 \\
0 \\
1 \\
4 \\
4 \\
0 \\
0 \\
4 \\
4 \\
4 \\
4\end{array}$ & $\begin{array}{l}0 \\
0 \\
0 \\
0 \\
0 \\
0 \\
0 \\
0 \\
2 \\
3 \\
0 \\
0 \\
3 \\
2 \\
2 \\
2\end{array}$ & $\begin{array}{l}4 \\
4 \\
4 \\
4 \\
4 \\
4 \\
4 \\
4 \\
4 \\
4 \\
0 \\
0 \\
4 \\
4 \\
4 \\
4\end{array}$ & $\begin{array}{l}2 \\
2 \\
2 \\
0 \\
0 \\
0 \\
0 \\
0 \\
4 \\
4 \\
0 \\
0 \\
2 \\
2 \\
1 \\
1\end{array}$ & $\begin{array}{l}0 \\
0 \\
0 \\
0 \\
0 \\
0 \\
0 \\
0 \\
2 \\
2 \\
0 \\
0 \\
1 \\
1 \\
0 \\
0\end{array}$ & $\begin{array}{l}4 \\
4 \\
4 \\
4 \\
4 \\
4 \\
4 \\
4 \\
4 \\
4 \\
0 \\
0 \\
4 \\
4 \\
4 \\
4\end{array}$ & $\begin{array}{l}0 \\
1 \\
0 \\
0 \\
0 \\
0 \\
0 \\
1 \\
4 \\
4 \\
0 \\
0 \\
4 \\
4 \\
4 \\
1\end{array}$ & $\begin{array}{l}0 \\
0 \\
0 \\
0 \\
0 \\
0 \\
0 \\
0 \\
4 \\
4 \\
0 \\
0 \\
1 \\
0 \\
1\end{array}$ \\
\hline
\end{tabular}

Table 2. Herbicidal activity of $N$-acylcaprolactams.

\begin{tabular}{|c|c|c|c|c|c|c|c|c|c|c|c|}
\hline \multirow[b]{2}{*}{$\begin{array}{c}\text { Compd. } \\
\text { No. }\end{array}$} & \multirow[b]{2}{*}{$\mathrm{R}$} & \multirow[b]{2}{*}{$\mathrm{X}$} & \multicolumn{3}{|c|}{ Radish } & \multicolumn{3}{|c|}{ Azuki-bean } & \multicolumn{3}{|c|}{ Rice } \\
\hline & & & $\begin{array}{r}100 \\
\times\end{array}$ & $\begin{array}{c}10 \\
100 \mathrm{ppm}\end{array}$ & 5 & $\begin{array}{l}100 \\
\times 1\end{array}$ & $\begin{array}{l}10 \\
00 \mathrm{p}\end{array}$ & 5 & $\begin{array}{r}100 \\
\times\end{array}$ & $\begin{array}{c}10 \\
.00 \mathrm{ppm}\end{array}$ & 5 \\
\hline $\begin{array}{l}17 \\
18 \\
19 \\
20 \\
21 \\
22 \\
23\end{array}$ & $\begin{array}{l}\mathrm{CH}_{3} \\
\mathrm{CH}_{3} \\
\mathrm{CH}_{3} \\
\mathrm{C}_{6} \mathrm{H}_{5} \\
\mathrm{C}_{6} \mathrm{H}_{4}-0-\mathrm{Cl} \\
\mathrm{C}_{6} \mathrm{H}_{4}-p-\mathrm{Cl} \\
\mathrm{C}_{6} \mathrm{H}_{4}-p-\mathrm{NO}_{2}\end{array}$ & $\begin{array}{l}\mathrm{H}_{2} \\
\mathrm{Cl}_{2} \\
\mathrm{H}_{1}, \mathrm{Cl} \\
\mathrm{H}_{2} \\
\mathrm{H}_{2} \\
\mathrm{H}_{2} \\
\mathrm{H}_{2}\end{array}$ & $\begin{array}{l}4 \\
4 \\
4 \\
2 \\
+ \\
+ \\
1\end{array}$ & $\begin{array}{l}0 \\
0 \\
0 \\
1 \\
+ \\
+ \\
+\end{array}$ & $\begin{array}{l}0 \\
0 \\
0 \\
0 \\
+ \\
+ \\
+\end{array}$ & $\begin{array}{l}2 \\
4 \\
4 \\
1 \\
+ \\
+ \\
+\end{array}$ & $\begin{array}{l}0 \\
0 \\
0 \\
0 \\
+ \\
+ \\
+\end{array}$ & $\begin{array}{l}0 \\
0 \\
0 \\
0 \\
+ \\
+ \\
+\end{array}$ & $\begin{array}{l}4 \\
4 \\
4 \\
1 \\
+ \\
+ \\
+\end{array}$ & $\begin{array}{l}0 \\
0 \\
0 \\
0 \\
+ \\
+ \\
+\end{array}$ & $\begin{array}{l}0 \\
0 \\
0 \\
0 \\
+ \\
+ \\
+\end{array}$ \\
\hline
\end{tabular}

$N$-Acetylation (Compd. 17) caused little effect on the herbicidal activity of caprolactam. Benzoylation (Compd. 20) caused rather decrease in the activity. Furthermore, it is interesting to note that the ring-substituted benzoyl derivatives (Compd. 21-23) somewhat stimulated germination. $\varepsilon$-Aminocaproic acid, i.e. the hydrolyzed product of caprolactam, also showed a similar germination stimulating property to all the tested plant species.

No post-emergence herbicidal activity was observed with any examined caprolactam derivatives.

Fungicidal activity has been found in some 2, 2-dichloro derivatives including 
Table 3. Herbicidal activity of amidine compounds and imino ethers derived from $\varepsilon$-caprolactam.<smiles>[R]C1=NCCCCC1</smiles>

\begin{tabular}{|c|c|c|c|c|c|c|c|c|c|c|}
\hline \multirow{2}{*}{$\begin{array}{c}\text { Compd. } \\
\text { No. }\end{array}$} & \multirow[b]{2}{*}{$\mathrm{R}$} & \multicolumn{3}{|c|}{ Radish } & \multicolumn{3}{|c|}{ Azuki-bean } & \multicolumn{3}{|c|}{ Rice } \\
\hline & & \multicolumn{3}{|c|}{$\times 100 \mathrm{ppm}$} & \multicolumn{3}{|c|}{$\times 100 \mathrm{ppm}$} & \multicolumn{3}{|c|}{$\times 100 \mathrm{ppm}$} \\
\hline $\begin{array}{l}24 \\
25 \\
26 \\
27 \\
28\end{array}$ & $\begin{array}{l}\mathrm{NHCH}_{3} \\
\mathrm{NHC}_{6} \mathrm{H}_{11} \\
\mathrm{NHC}_{6} \mathrm{H}_{4}-m-\mathrm{CH}_{3} \\
\mathrm{NHC}_{6} \mathrm{H}_{4}-0-\mathrm{OCH} \mathrm{H}_{3} \\
\mathrm{NHC}_{6} \mathrm{H}_{4}-m-\mathrm{Cl}\end{array}$ & $\begin{array}{l}0 \\
4 \\
4 \\
4 \\
4\end{array}$ & $\begin{array}{l}0 \\
0 \\
0 \\
0 \\
1\end{array}$ & $\begin{array}{l}0 \\
0 \\
0 \\
0 \\
0\end{array}$ & $\begin{array}{l}0 \\
4 \\
4 \\
4 \\
4\end{array}$ & $\begin{array}{l}0 \\
0 \\
0 \\
0 \\
0\end{array}$ & $\begin{array}{l}0 \\
0 \\
0 \\
0 \\
0\end{array}$ & $\begin{array}{l}0 \\
4 \\
4 \\
4 \\
4\end{array}$ & $\begin{array}{l}0 \\
0 \\
0 \\
0 \\
0\end{array}$ & $\begin{array}{l}0 \\
0 \\
0 \\
0 \\
0\end{array}$ \\
\hline 29 & $\mathrm{NH}$ & 4 & 1 & 0 & 4 & 0 & 0 & 4 & 1 & 1 \\
\hline $\begin{array}{l}30 \\
31\end{array}$ & $\begin{array}{l}\mathrm{OCH}_{3} \\
\mathrm{OC}_{2} \mathrm{H}_{5}\end{array}$ & $\begin{array}{l}1 \\
1\end{array}$ & $\begin{array}{l}0 \\
0\end{array}$ & $\begin{array}{l}0 \\
0\end{array}$ & $\begin{array}{l}1 \\
1\end{array}$ & $\begin{array}{l}0 \\
0\end{array}$ & $\begin{array}{l}0 \\
0\end{array}$ & $\begin{array}{l}0 \\
0\end{array}$ & $\begin{array}{l}0 \\
0\end{array}$ & $\begin{array}{l}0 \\
0\end{array}$ \\
\hline
\end{tabular}

Compd. 3, 15, and 16. They are effective to control Pyricularia oryzae, Fusarium oxysporum, and Rhizoctonia vagum (Oshima et al., 1972).

A part of this report was presented to the West Japan District Annual Meeting of Agricultural Chemical Society of Japan at Kagoshima, 1965.

\section{REFERENCES}

Baszynski, T. and L. Rejniak 1958 Caprolactam as a growth factor in plants. Experiments with Pisum sativum seedlings. Acta Soc. Botan. Polon., 27 : 249-261; Chem. Abstr., 53: 8309 (1959)

Benson, R. E. and T. L. Cairus 1948 Chemical reactions of caprolactam. J. Am. Chem. Soc., $70: 2115-2118$

Marvel, C. S. and W. W. Moyer 1957 Some derivatives of $\varepsilon$-caprolactam. J. Org. Chem., 22: $1065-1067$

Oshima, Y., S. Yamazaki, H. Araki, M. Kobayashi, H. Kaku, Y. Takabi and K. Nakagami 1972 Agricultural fungicides. Japanese Patent, 47-37535

Wilson, H. F., D. H. McRae and B. M. Vittimberga 1964 N-(Halophenyl)lactams. French Patent, 1,349,517; Chem. Abstr., 60 : 13197

Wineman, R. J., E. T. Hsu and C. E. Anagnostopoulos $1958 \alpha$-Halogenation products of $\varepsilon-$ caprolactam and their transformation to DL-lysine. J. Am. Chem. Soc., 80:6233-6237 